\title{
La influencia de los procesos de barrismo social en el Barón Rojo Sur y la Fundación Un Distrito en Paz: Una nueva perspectiva de Aguante
}

\section{Juan Sebastián Castillo Castro ${ }^{1}$ \\ juansebastiancastillocastro@gmail.com}

\author{
Universidad Icesi
}

Artículo de investigación recibido el 01/02/2019

y aprobado el 02/03/2019

Cómo citar este artículo:

Castillo Castro, J. (2019). La influencia de los procesos de barrismo social en el Barón Rojo Sur y la Fundación Un Distrito en Paz: una nueva perspectiva de aguante. TransPasando Fronteras, (13). doi: 10.18046/retf.i13.3400

\footnotetext{
${ }^{1}$ Estudiante de Sociología y Ciencia Política con Énfasis en Relaciones Internaciona-
} les de la Universidad Icesi. 


\section{Resumen}

El presente artículo analiza el caso de Barón Rojo Sur (en adelante B.R.S) y de la Fundación Un Distrito en Paz (en adelante FUNDP) en clave del barrismo social, pues a partir de las acciones que se inscriben en este tipo de barrismo, se abre un espacio para repensar la noción de Aguante desarrollada por teóricos argentinos. La investigación se realizó con metodología cualitativa que fue aplicada a integrantes de la barra y de la fundación. Entre los principales hallazgos se encontró que, para el caso particular de B.R.S. los procesos de barrismo social van de la mano de formas de ciudadanía activa y participativa y de procesos sociales y políticos. Además, el proceso realizado por la Fundación Un Distrito en Paz, que es liderado por integrantes de la barra, evidencia la consolidación y formalización de un proceso organizativo de barrismo social. Este tipo de procesos no podrían ser explicados, completamente, por la visión tradicional que se tiene del aguante. Razón por la cual, se propone un cambio en la perspectiva para dar una mirada analitica desde el Barrismo Social.

Palabras clave: Fútbol; Barrismo social; Barras; Barón Rojo Sur Aguante 


\section{The influence of Barrismo Social process in Barón Rojo Sur y la Fundación Un Distrito en Paz: New pespective of Aguante}

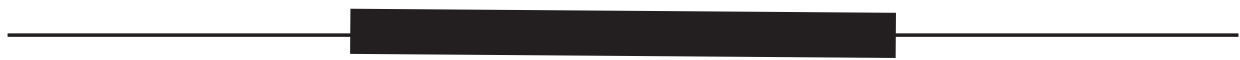

\section{Abstract}

This article analyzes the case of Barón Rojo Sur (BRS), and the Fundación un Distrito Para la Paz (FUDNP) in relation to the Barrismo Social, since the actions within this type of barrismo opened a space to rethink the notion of aguante made by argentine theorists. The research was conducted with qualitative methodology that was applied to BRS soccer supporters and the ONG. Among key findings it was found that, in the case of BRS, the processes of barrismo social work hand in hand with forms of active and participative citizenship. Moreover, it is tied to social and political processes. So, the process carried out by the Fundación un Distrito en Paz, which is led by members of the BRS football supporters, demonstrates the consolidation and formalization of an organizational process of Barrismo Social. This type of process could not be explained completely by the traditional view of aguante. Therefore, a change in the perspective is proposed to give a analitic view from the Barrismo Social.

Palabras clave: Soccer; Barrismo Social; Hooligans; Barón Rojo Sur; Aguante; Violence. 


\section{Introducción}

En la década del 90 surgieron barras en la mayoría de los equipos de futbol profesionales del país (Jairo Clavijo 2011). Para el caso del equipo América de Cali, la barra que actualmente lo acompaña es Barón Rojo Sur (en Adelante B.R.S o B.R), la cual se empezó a conformar durante los años 90's y se llamó inicialmente, furia roja, hasta que en 1997 se consolida en lo que actualmente es Barón Rojo Sur.

El surgimiento de estos colectivos replanteó en la opinión pública, la forma de entender los hechos de violencia o criminalidad alrededor del fútbol, pues pasaron de ser concebidos como enfrentamientos entre particulares a una violencia ejercida desde un grupo organizado. Si bien, el conflicto físico no está cerca de ser la actividad principal, ni el objetivo del Barón Rojo Sur u otras barras de Colombia sí ha sido suficiente para construir a través de los medios de comunicación y de la sociedad en general un arquetipo violento hacia la barra y hacia sus integrantes.

Los principales estudios a nivel de américa latina sobre barras han estado centrados en explicar la violencia que se genera en estos colectivos a partir del concepto de Aguante (Garriga, 2006) y de su organización burocrática a través de la noción de Hinchadas Organizadas (Máximo, 2014). Ambos conceptos serán desarrollados más adelante en este artículo y confrontados con la realidad de Barón Rojo Sur.

Cabe resaltar que la preocupación por la violencia de las barras, también ha estado en el ojo de los barristas colombianos, quienes en el año 2007 convocan la conformación del Colectivo Barrista Colombiano, en el que se identifica que, dentro del accionar de la barra, hay una serie de prácticas positivas que pueden 
contribuir a mitigar los episodios de violencia. Posteriormente, representantes de diferentes barras en colaboración con la fundación Juan Manuel Bermúdez Nieto, y entidades gubernamentales, conforman una mesa de trabajo de trabajo que sienta las bases para la elaboración de una política pública que incluya las diversas formas de barrismo (Arroyo, 2014). A raíz de esto, surgen diferentes documentos públicos que reconocen formalmente el concepto Barrismo Social, que se desarrollará, más adelante, como una contra propuesta al concepto de Aguante, y que se pretende pueda ofrecer una nueva perspectiva de análisis para explicar la realidad de los barristas colombianos.

En B.R.S emergen distintos procesos sociales y políticos, entre ellos la Fundación Un Distrito en Paz (en adelante FUNDP), la cual será presentada como un caso de análisis útil para avanzar en la literatura sobre Barras, pues ofrece un ejemplo de consolidación y formalización de los procesos que surgieron desde la barra Barón Rojo Sur.

Para ello se diseñaron herramientas que permitieran conocer historias, opiniones e ideas, y que dieran cuenta de la complejidad y las dificultades que se dieron al desarrollar los procesos dentro de la barra y la Fundación Un Distrito en Paz. Las herramientas utilizadas fueron entrevistas semi-estructuradas, que se realizaron a barristas, líderes del B.R.S. y líderes de los procesos de barrismo social. Las preguntas se centraron, en su experiencia o conocimiento sobre el barrismo social y, en el caso de los integrantes de la fundación, en su experiencia consolidando el proceso.

El presente documento se divide en cuatro secciones: (i) El marco interpretativo, en el que se desglosan los principales hallazgos teóricos sobre barras, que pueden ser sintetizados en el concepto de Aguante y de Hinchadas Organizadas. Posteriormente, (ii) se 
analiza las experiencias de integrantes de Barón Rojo, pues la barra ha dado lugar al surgimiento y a la construcción de procesos sociales, políticos y educativos que ponen en manifiesto la necesidad de avanzar en la literatura tradicional con la que ha sido analizado el fenómeno de las barras. Luego, (iii) se describe el caso de la Fundación Un Distrito en Paz cuyo surgimiento se da en Barón Rojo y se ha convertido en un referente, por su consolidación organizacional. Dicho proceso también arroja luces sobre la complejidad del contexto en el que se encuentra la barra, que resulta enriquecedor para la comprensión del barrismo social y de la dinámica de la barra como Barón Rojo Sur. Finalmente, (iv) se realiza una sucinta recopilación de reflexiones en torno al barrismo social, cuyo reconocimiento legal y normativo ofreció espacios para que se consolidaran procesos como los descritos de B.R.S. y FUNDP. Ante esto se propone el Barrismo Social como un concepto desde el cual se puede analizar las dinámicas de las barras que, a diferencia del Aguante, reconoce procesos de participación política y de inclusión social que se dan dentro de las barras.

\section{(i) Marco Interpretativo}

En un principio, teóricos como Norbert Elías y Eric Dunning (1996) realizaron estudios sobre el Hooliganismo, en los cuales concluían que la violencia que se desataba en los estadios podía explicarse desde una noción de clase y del relajamiento de los controles emocionales, que se produce al estar en una masa (Elías y Dunning, 1996).

Sin embargo, en Argentina, se reconoció que las diferencias entre el aficionado europeo y el latino, ameritaba elaborar nuevos aportes teóricos que comprendieran las barras de fútbol argentinas 


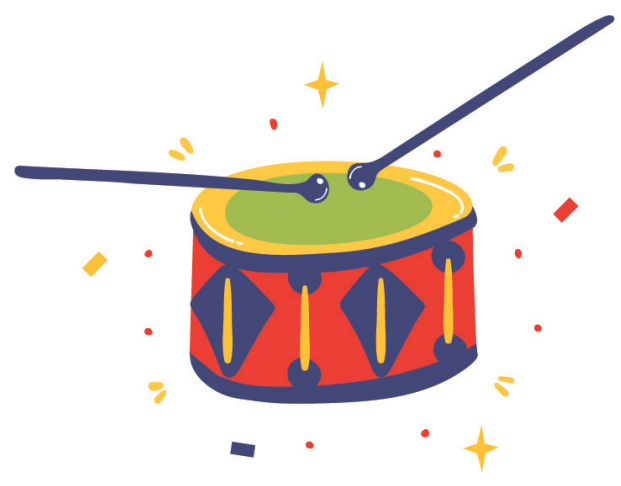

"Los barristas se distinguen de los otros

hinchas por una serie de elementos de autoreconocimiento como la fidelidad al equipo, el fervor como se vive el fútbol y la hermandad con los otros integrantes de la barra." en su particularidad. Es por ello que para el caso latinoamericano los barristas y la barra se vuelven sujetos de investigación, porque lo que interesaba principalmente era poder comprender la barra como un fenómeno social y no como un variable, que explicara el desenlace de la violencia.

Los barristas se distinguen de los otros hinchas por una serie de elementos de auto-reconocimiento como la fidelidad al equipo, el fervor como se vive el fútbol y la hermandad con los otros integrantes de la barra. Su identidad es el motor de una serie de prácticas como los canticos, los saltos, e incluso de manifestaciones violentas que en algunos casos están dispuestos a realizar por el equipo al que alientan (Garriga, 2006).

Al igual que en Colombia, en donde se ha consumado un estereotipo agresivo alrededor de las barras, en países como Argentina, Chile y Brasil, la principal preocupación de la academia, a la hora de realizar estudios de 
barras fue, explicar los orígenes y las motivaciones de las prácticas violentas que sucedían en torno al fútbol. Entre los hallazgos se encontró que, de manera particular, la violencia que se produce en la barra tiene legitimidad a través del "Aguante". Esté representa para los barristas el bien simbólico principal a la hora de construir y mantener la identidad, pues hace parte de un sistema de prestigio y honor necesario para la construcción de la identidad colectiva de la hinchada (Garriga, 2006).

El Aguante es realmente importante para la identidad del barrista, por ende, es necesario dar cuenta que se posee. Para tenerlo no basta con decirlo, sino que hay que demostrarlo. El Aguante se prueba a través del cuerpo, puede ser saltando 90 minutos en un partido, o defendiendo los colores del equipo, en una pelea con la policía o con rivales de otra barra.

Uno de los incentivos de demostrar el Aguante, es escalar dentro de la jerarquía de reputación de la barra, cuyo ascenso otorga, a los barristas, reconocimiento por parte de sus pares dentro de la barra y de actores externos. Además, la relación con actores por fuera de la barra genera un insumo de capital social y privilegios, que de otra forma costaría mucho trabajo tener, pues una población importante de los barristas pertenece a contextos en donde carecen de este reconocimiento social (Cabrera, Czesli y Garriga, 2016).

Adicionalmente, otro de los elementos que distingue a los Barra de los hinchas tradicionales, es que pertenecen y se reconocen dentro de un colectivo que cuenta con una estructura organizacional. Por ello Carlos A. Máximo Pimienta (2013) plantea las distinciones entre las barras y el resto de los hinchas a través de la organización. Para el autor las hinchadas organizadas "constituyen sus acciones a partir de estructuras burocráticas, valorizadas por situaciones de pertenencia fomentadas por símbolos, significados, significantes y 
significaciones que determinan identificaciones visuales por medio de la creación de la marca del hincha" (Máximo, 2014, pp. 296). De igual forma, la estructura organizacional se mantiene gracias a que existen normas, roles formales, funciones, estatutos, sedes, reuniones e interacción social.

En este tipo de colectivos, la cohesión social se hace posible por el entramado de elementos que hacen parte de su identidad, los significados compartidos y las manifestaciones "por", "en", "de" y "entre" espacios y territorios (Máximo, 2014). No obstante, la estructura se entrelaza con las manifestaciones de violencia, dando lugar a la institucionalización de modos de organización, estrategias y tácticas de defensa y de acción en los enfrentamientos con aquellos que son considerados enemigos (Máximo, 2014).

En suma, se podría decir como elementos explicativos de las barras se encuentra, por un lado, el nivel de organización de las barras, que las diferencia de los demás aficionados, en cuanto a su estructura jerárquica, funciones, reuniones, etc... Por otro lado, está el Aguante, que se refiere a todo el sistema en el que se marcan las acciones del barrista, que incluye la jerarquía, la reputación, el honor y los discursos que son fundamentales para la identidad barrista. Respecto al último, es importante tener en cuenta, que en el repertorio de acciones para ganar prestigio y reputación se encuentran prácticas violentas y de confrontación.

De la misma manera, probar el aguante a través de la violencia y escalar dentro de la escala de reputación de la barra, otorga a los hinchas reconocimiento por parte de sus pares dentro de la barra y de actores externos (Cabrera et al. 2016). 


\section{Sobre Barón Rojo Sur y Santiago de Cali (sede del equipo América de Cali)}

Ahora bien, para el caso de Cali, Joanna Rojas (2013) plantea que la violencia de las barras de la ciudad de Cali, se produce, en gran parte, porque muchos de los elementos identitarios de los barristas tienen una fuerte carga simbólica, como, por ejemplo, territorios, tatuajes o atuendos, que son fácilmente identificados por barras rivales, y usados o transgredidos con el fin ofender o cuestionar la identidad rival (Castro, 2010).

Para distinguir a los Barra y a B.R.S. del resto de los hinchas que van al estadio, es menester hablar de los colores, espacios, territorios, formas de vestir, prácticas lúdicas (cantos, orquestas y coreografías) y de la rivalidad con otros grupos similares u otras hinchadas organizadas. Incluso, también se pueden distinguir por la conjunción de elementos más complejos como la masculinidad, virilidad, el fervor y el sentido de pertenencia (Máximo, 2014). No obstante, aunque no es el objetivo de este trabajo, es pertinente aclarar que otro de los elementos que hacen parte del entramado complejo de la barra, son las feminidades, pues en la barra hay un número considerable de mujeres que la integran, cuyas acciones y comportamientos son determinantes en el accionar de la barra.

\section{(ii) Cambio de perspectiva de Aguante: experiencia Barón Rojo Sur.}

Uno de los principales hallazgos que se lograron en la recolección de información es que B.R.S. se puede entender como una fotografía social, pues congela durante 90 minutos un grueso de la población supremamente heterogéneo, en el que hay individuos de distintos estratos, zonas de la ciudad, del país, de diferentes colores de piel, género, clase y contexto. Es decir que la barra no es 
una unidad homogénea, sino que dada su amplia diversidad debe ser abordada como un objeto de estudio dinámico, sobre el cual sentar un marco rígido de análisis resulta insuficiente.

A pesar de su amplia diversidad hay elementos en común entre los individuos que finalmente dan la cohesión necesaria para la existencia de Barón Rojo. Partido tras partido, asistentes al estadio y televidentes han sido testigos que, en La Sur ${ }^{2}$ se presenta un entramado de realidades unidas por una identidad compartida que bajo una serie de símbolos y códigos dan forma al Barón Rojo.

Es decir, la identidad barrista agrupa diversidad de individuos que pueden ser entendidos como una muestra de la ciudad, razón por la cual, así como en la ciudad hay problemáticas de crimen, homicidios, seguridad, y drogas, en la barra también se presentan este tipo de acciones. Lo anterior, no implica que las acciones de violencia y criminalidad sean endógenas a los barristas, ni se comentan, necesariamente, de manera organizada bajo el nombre de la barra, sino que su origen se encuentra en elementos como el contexto de donde provienen los integrantes.

Esto replantea la visión tradicional de abordar la violencia en las barras, dejándola de ver como un problema social propio del estadio, un problema social endémico del colectivo, o un problema social del fútbol, y abre la posibilidad para considerarlo un problema social de ciudad.

Efectivamente, no se puede negar que la violencia haya sido usada por algunos integrantes de B.R.S. para ganar reputación y reconocimiento por parte de sus pares.

2 Tribuna Sur del estadio Olímpico Pascual Guerrero donde suele ubicarse Barón Rojo Sur. 
"Para ser un referente de la barra se gana también por medio de la violencia, entonces yo soy una persona, un ejemplo, el que más he robado trapos, cada viaje me meto donde los hinchas rivales y les robo un trapo, cada rato me meto a atacar a los rivales y los correteo, o el que cuando aquí no me quieren hacer caso, me paro con el que sea. Ese es el que demuestra ese poder por medio de la violencia de los golpes, de hablar fuerte. Entonces ahí va creando ese respeto, entonces ya ese peladito, que es cólico y cree, dice ese es el capo, uff. Es una forma en la que, no solo en esta barra sino en todas las barras, el que llega a ser referente se gana su respeto por eso." (Héctor, comunicación personal, 16 de agosto de 2018).

Sin embargo, dada la heterogeneidad de la barra y la diversidad de pensamientos e ideas que confluyen en ésta, muchos de los líderes se encuentran en desacuerdo con este tipo de acciones y creen que, aunque es producto del contexto en el que se encuentran, la barra puede ser el lugar en donde se neutralicen este tipo de conductas, cumpliendo con una función política y social.

"Uno sabe, que acá, vos podés encontrar una persona que se fue a estudiar, podes encontrar una persona que no ha tenido las mismas oportunidades, pero no es de dejarlo a un lado, no es de hacerlo a un lado y decirle, 'no, vos no podés ser parte de esto', es integrar a todos $y$ todos tenemos una facultad, todos tenemos algo para lo que servimos, todos tenemos algo que nos caracteriza y nos dice esta es tu habilidad, y ahí es donde se tiene que explotar,

“¿Si me entendés?" (Lideresa Barón Rojo Sur, comunicación personal, 19 de agosto de 2018).

"Hay otros que viven en la calle, o no tienen oportunidades para estudiar, o tienen muchas dificultades en sus casas y sus barrios, entonces algunos líderes nos damos ese papel de poder trabajar 
en esos espacios y brindarles educación, formaciones en varias áreas de acuerdo a lo que ellos quieran explorar de acuerdo a sus perfiles, y esa es la manera como trabajamos nosotros, pues algunos nos responsabilizamos de eso, otros no, pero ahí está la esencia de Barón Rojo Sur." (Bryan, comunicación personal, 19 de agosto de 2018)

Este tipo de acciones demuestran que los líderes de la barra, no están simplemente coaccionados bajo las lógicas del discurso del Aguante tradicional (es decir, el Aguante trabajado por autores argentinos), sino que reconocen la función social que cumple B.R.S para la sociedad, y perciben que los problemas que se dan son de corte estructural. Ante ello, los barristas entrevistados proponen trabajar desde la barra para transformar la consciencia y la forma de pensar de las otras personas. Asimismo, se pretende direccionar sus habilidades, para ser útiles tanto en la barra como fuera de ella. También, se apunta a crear una consciencia política en los Barra que integran Barón rojo.

"Nosotros desde los inicios del Barón Rojo Sur, desde la fundación, desde el 97, Barón Rojo Sur se une a los ideales de las luchas sociales y pues hasta el día de hoy tratamos de mantener nuestra vinculación con la problemática social” (Brayan, comunicación personal, octubre 10 de 2018).

"Hay una responsabilidad que nosotros tenemos directamente porque nosotros dentro de la tribuna que yo mencionaba anteriormente, hay muchas culturas, subculturas, diversidad de género, tenemos pelados desde los 12, hasta personas con 40 - 50 años, nosotros lo que hacemos es mirar la problemática que se vive al interior de la barra, y pues tratar de trabajar como el camino al pro de un cambio" (Brayan, comunicación personal, octubre 10 de 2018). 
Esto permite ver que Barón Rojo Sur ha servido de plataforma para pensar de manera organizada formas de construcción de subjetividad política para los actuales integrantes de la barra, utilizando como insumo la identidad colectiva que tienen como Barras. De esta manera, valdría la pena preguntarse sí Barón Rojo puede llegar a cumplir una función social y política en la ciudad.

No obstante, es pertinente resaltar que, por la misma heterogeneidad de la barra, se dificulta el desarrollo de las iniciativas de corte político y social, ya que, así como se encuentran personas con el interés de mejorar la calidad de vida de los demás y de promover el aguante social, es probable que también se encuentren integrantes que solo van a consumir o alentar y no consideren que este tipo de iniciativas tengan que ver con la institución, ni con el ejercicio de ser Barra.

\section{Nueva propuesta de análisis: barrismo social.}

¿Cuál puede ser una mirada adecuada para aquellas acciones generadas por las barras con significado y contenido político? Es probable que abordar a las barras colombianas desde la mirada del aguante, que ha sido trabajado en otros países, resulte en un intento fallido por importar un concepto que no explica en su totalidad las dinámicas de los barristas.

La conformación en el año 2003 del Colectivo Barrista Colombiano, que contaría con la presencia de 18 líderes de las 21 barras del país, y se encargarían de trabajar sobre los problemas de convivencia y violencia que giraban en torno a las barras, fue el principio de la construcción colectiva del concepto, barrismo social. Con el apoyo de la Fundación Juan Manuel Bermúdez Nieto (en adelante FJMBN) y el gobierno nacional, se sentaron 
las bases para la construcción de una política pública sobre barras, que no se centrara solamente en el ejercicio de la seguridad desde el aumento de la fuerza pública (Arroyo, 2014).

La presión ejercida por el colectivo barrista sobre las entidades gubernamentales tuvo sus primeros resultados al lograr introducir la idea de barrismo social en la ley 1270 de 2009, como una alternativa que debe ser promovida por las recientemente creadas por esa misma ley, las Comisiones Locales de Seguridad, Comodidad y Convivencia en el Fútbol (Ley 1270, 2009). Adicionalmente, el decreto 1007 especificaría que por barrismo social se entiende:

"Acciones encaminadas a redimensionar las formas de expresión y las prácticas de los integrantes de las barras de fútbol que inciden negativamente en los ámbitos individual, comunitario y colectivo, y de potenciar los aspectos positivos que de la esencia del barrismo deben rescatarse. Esta propuesta se fundamenta en procesos formativos tales como el diálogo de saberes, que recogen valores sociales, normas, creencias, ideales y sentimientos, y les permiten a los barristas resignificar la realidad que los sumerge en su pasión por el mundo del fútbol, y a asumir así su identidad como sujetos sociales y participativos." (Decreto 1007, 2012).

Posteriormente, el Plan Decenal de Seguridad Comodidad y Convivencia en el Fútbol (en adelante PDSCCF) retoma la definición presentada anteriormente, y se propone como objetivo general, el fortalecimiento del modelo de barrismo social, propuesto en el decreto 1007, a través de

"El desarrollo de liderazgos positivos, la consolidación de sus estructuras y estrategias de planeación interna, y el diseño e implementación de proyectos y programas que permitan potenciar el crecimiento individual y grupal de sus miembros. Se trata de que 
sean ciudadanos activos que inciden y participan positivamente en espacios sociales políticos en los que desarrollan su actividad: barrio, comunidad y ciudad" (Ministerio del interior, 2014)

Dada la insuficiencia del Aguante para explicar las acciones descritas anteriormente, el concepto de barrismo social, que ha sido construido por parte de los barristas a nivel nacional, puede dar luces sobre nuevas formas de comprender a las barras.

\section{¿Acaso la violencia puede ser política?}

Es importante resaltar que, partir de la premisa de que, el barrismo social a través de la creación de consciencia política, tiene como efecto la reducción de manifestaciones violentas, puede tener una limitación. Ya que, los enfrentamientos con la policía, además de ser generados por la necesidad de demostrar el Aguante, pueden estar respaldados por un ejercicio de resistencia civil, ante los casos donde se considera que el Estado ejerce represión estatal. Por ejemplo, cuando se percibe en la policía una actitud bélica o humillante, cuando se le niega a los barristas la posibilidad de entrar a otra ciudad, o cuando se prohíben las reuniones en lugares públicos.

Cabe resaltar que, el Artículo 37 de la Constitución Política de Colombia afirma que, "toda parte del pueblo puede reunirse y manifestarse pública y pacíficamente. Sólo la ley podrá establecer de manera expresa los casos en los cuales se podrá limitar el ejercicio de este derecho" (Const.,1991, art 37).

Esto también lo identifican como una forma de represión, pues algunos sectores de la barra consideran que B.R.S. tiene una serie de posturas políticas, que corresponden su carácter popular, y que pueden ir en contra del Estado. 
"Muchos casos donde esta violencia se ha visto, desde esa represión, y más en el BR, que el BR ha sido una barra rebelde y que sea como sea siempre tiene una postura, de siempre protestar cuando hay posturas políticas [...] por eso siempre ha recibido ese tipo de represión" (Héctor, comunicación personal, 16 de agosto de 2018).

"Porque todos somos actores de esta nación, y pues los sectores más vulnerables están en las barras populares, entonces yo creo que eso es uno de los elementos más importantes para que nosotros nos apropiemos de la lucha, por eso salimos a las calles a protestar" (Brayan, comunicación personal, octubre 10 de 2018).

Ante este escenario, en la barra se conforma un comité de derechos humanos Ilamado Joan Sebastián Tenorio Bernal, en honor a un integrante de la barra que, presuntamente, fue asesinado en carretera"

"El comité de DDHH surge de la necesidad de velar por los derechos de nuestros compañeros en los viajes" (Líder comité DDHH, comunicación personal, octubre 10 de 2018).

"Las personas que van a las barras, no solamente en barón rojo sur, sufren mucha problemática, no solamente en las ciudades en estadios locales, sino también en los estadios a nivel nacional, cuando van a viajar para ver a sus equipos, y en las carreteras hay muchos atropella, atropella mucho la policía, entonces pues es una manera de resistencia y de lucha." (Líder comité DDHH, comunicación personal, octubre 10 de 2018).

En cierta medida, la postura política que demuestran algunos de los miembros de Barón Rojo Sur, entre ellos líderes de la barra, ofrece indicios para considerar que la reacción hacía la policía, no 
siempre corresponde a lo que Garriga (2006) considera podría ser, una cuestión del Aguante como parte del sistema de prestigio y honor, sino que abre el espectro para incorporar la posibilidad de entender esta reacción, desde el Barrismo social (Garriga, 2006). Por polémico que parezca, son acciones que se dan en clave de un proceso de lucha y de resistencia ante abusos de la fuerza pública, referenciados como represión, hacía la postura política que se ha construido en la barra como colectivo.

De manera similar, las autoras Londoño y Pinilla (2009) plantearon que, contrario a la postura de autoras clásicas como Hannah Arendt, en la que la política está ligada los factores discursivos y concertativos, la violencia realizada por los Barras de Holocausto Norte se puede inscribir en la dimensión política, pues está configurada en una lucha por la integridad física y cultural de los barristas (Londoño y Pinilla, 2009).

Asimismo, en la aproximación a Barón Rojo se evidenció que, además de los elementos que son similares entre ambas barras, también se pueden inscribir las prácticas de la barra en la dimensión política, dado que se encuentra relacionadas con la disipación del prejuicio criminalizante que hay sobre los barristas. En ese sentido, los enfrentamientos con la policía en varias ocasiones son vistos como formas de resistir ante la falta de reconocimiento y al prejuicio criminalizante que tiene la fuerza pública y que se trasmite en medios de comunicación

Lo anterior, permite ver cómo B.R.S ha dado lugar a la consolidación de procesos de carácter político y social, que pueden ser explicados desde la noción de Barrismo Social. Sin embargo, el reconocimiento de Barrismo Social en la ley, tuvo como consecuencia la promoción de este tipo de acciones. Por tal razón, es pertinente para el presente trabajo dar cuenta de la experiencia de la 
Fundación un Distrito en Paz, que surge en los últimos años, y que permite ver un impacto que ha tenido el trabajo del colectivo barrista y el reconocimiento normativo del Barrismo Social.

\section{(iii) Caso: Fundación un Distrito en Paz}

La Fundación Un Distrito En Paz (En adelante FUNDP) surge en el año 2016, lastimosamente, producto del asesinato de dos de los miembros del Bloque Distrito Popular de Barón Rojo Sur. Estos dos jóvenes asesinados, habían marcado un precedente dentro de la barra, pues, posterior al proceso de reconocimiento legal del barrismo social, ellos se encargaron de promoverlo dentro de la barra.

"Por todo el tema de barrismo social, la barra avanzó muchísimo, la barra... el cambio que tuvo en esa dirigencia, yo creo que fue visible en toda la ciudad, una barra diferente, una barra donde los jóvenes ya no se veían consumidos por las drogas, sino que realmente había aguante, vos ya entrabas a la tribuna y sentías el aguante, los 90 minutos, cambio mucho la estética de la barra, era una barra que se preocupaba por hacer salidas espectaculares, sacar trapos espectaculares, tapa-tribunas, realmente una barra de proyectos. Él tenía un grupo de trabajo, y a ellos los empezaron a amenazar" (Héctor, comunicación personal, 16 de agosto de 2018).

Dada la complejidad de la barra y las dinámicas de criminalidad que existen en ella, ambos jóvenes fueron asesinados. A pesar, de los odios que quedaron luego de las muertes, se marcó un precedente y se dejó como legado la idea de que el Barón Rojo, podía ser diferente y apuntar hacía la función social y política de la barra.

"La verdad la gente de este bloque querían acabar con todo, querían acabar con la barra, teníamos con qué, pero tomamos 
la decisión de decir no, si ellos murieron fue porque realmente lucharon por algo diferente para la barra y lo demostraron con hechos" (Héctor, comunicación personal, 16 de agosto de 2018).

Así, quienes habían estado tras la sombra de ellos en los procesos de transformación de la barra, deciden continuar con el legado que les habían dejado sus referentes, y crean la FUNDP. Desde antes de crear la fundación, se había identificado que las problemáticas de la barra que se manifiestan durante los partidos, no se originan exclusivamente en ese momento, sino que son problemas que vienen desde los territorios.

"Entonces nos empezamos a apropiar de espacios como esta plazoleta, que acá se hacen ciclovías, de las bibliotecas que hay acá. Empezamos a ir a las fronteras invisibles, que hay acá en el distrito con la pedagogía del fútbol, a cambiar todas esas realidades" (Héctor, comunicación personal, 16 de agosto de 2018).

Intervenir los territorios no solamente apunta a promover formas de barrismo positivas, sino a reducir en los jóvenes, niños y niñas, que posiblemente pueden ser futuros barristas, las condiciones de marginalidad, desigualdad y oportunidades, fomentando procesos deportivos y culturales que les muestren alternativas a la criminalidad y la violencia. Lo anterior, se debe a que, si no se trabaja la violencia en las barras como un fenómeno integral, este va a continuar materializándose en el estadio.

En la misma vía, en la que FUNDP empezó a dirigir sus intervenciones, el teórico argentino José Garriga Zucal (2006) desarrolla el texto Acá es así, en el que describe el papel del barrio para el desenlace de la violencia de los barristas, porque, en los casos en donde ésta se remonta a unas condiciones de marginalidad y exclusión, se adquieren nuevos pilares para legitimar prácticas de violencia (Garriga, 2006). 
"Por un lado, una concepción del espacio 'marginal' o pobre vinculado a la violencia, que define como particularidad de la pobreza a la violencia. Por el otro lado, una recuperación del 'pasado' (recorte parcial y tendencioso) que tiene como objeto demostrar que desde hace muchos años la violencia es la característica que define la forma de relacionarse en estos lugares." (Garriga, 2006, p.105)

El territorio donde se ubica el Bloque Distrito Popular, se llama, por divisiones político- administrativas, Distrito de Aguablanca. Este territorio cuenta con una serie de condiciones que hacen difícil la obtención de condiciones dignas, además de una alta tasa de homicidios. En los proyectos de barrismo social realizados en estos lugares, se encontraron con jóvenes que nunca había tenido la posibilidad de salir del distrito, así que, al finalizar un proceso pedagógico sobre las barras, se les llevó al Estadio, para que conocieran otra realidad y se enamoraran -así sea poco- de los valores del fútbol. Por tanto, en los jóvenes que provienen de estos contextos, hay una necesidad de que el barrismo social se realice considerando como un elemento clave al territorio, debido a que en él puede haber elementos que promuevan la violencia.

En esta medida, FUNDP se ha encargado de resaltar el valor del territorio y de marcar la territorialidad de este, sin recurrir a prácticas violentas. Por ejemplo, en la plaza donde se reúne la barra, se pintaron murales en honor a uno de los jóvenes asesinados, y también, en conjunto con un colegio aledaño a la plaza, gestionaron una jornada de recolección de escombros para recuperar el espacio público. Este tipo de iniciativas han permitido que sean reconocidos dentro de la comunidad a la que pertenecen y que puedan usar el espacio público para reuniones de bloque, sin tener como enemigo a la comunidad. Por otro lado, FUNDP se ha dedicado también a dictar diversos talleres y ejercicios de pedagogía, 
en relación con las dinámicas de la barra y la desestigmatización de ésta, a través de la creación de la escuela futbolera. Este proyecto representa una apuesta de la fundación por desmitificar todos aquellos imaginarios sobre los barristas, en los jóvenes del Distrito, y también, en la promoción de los valores del barrismo que no están relacionados con la violencia.

"Primero que todo para ser barra se necesita saber la historia del equipo, historia de América de Cali, de cómo nace el fútbol, los movimientos barras en el mundo, como llega eso a Sudamérica a Colombia, como barras que ya han evolucionado mucho llegan a incidir en temas políticos y sociales de su país" (Héctor, comunicación personal, 16 de agosto de 2018).

Este tipo de apuestas son posibles porque ya ha habido una reconfiguración del discurso tradicional de lo que implica ser barrista. La visión con la que FUNDP ofrece los talleres

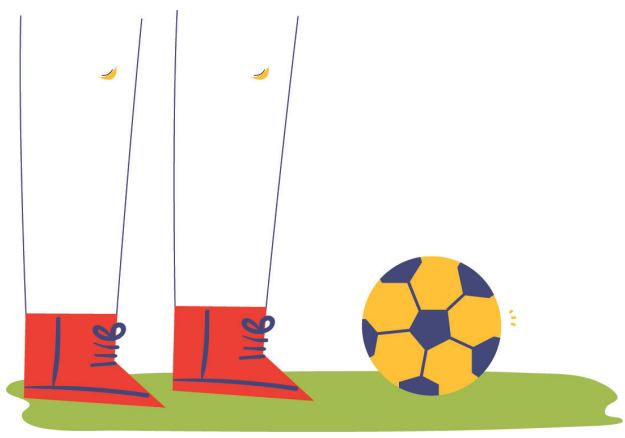

"Primero que todo para ser barra se necesita saber Ia historia del equipo, historia de América de Cali, de cómo nace el fútbol, los movimientos barras en el mundo, como llega eso a Sudamérica a Colombia..." 
es, promover que lo necesario para ser parte de una barra, vaya más allá de las rivalidades con otras personas e instituciones y de acciones agresivas como fuente de reputación, e implique ser conscientes del rol que se tiene siendo barrista y del papel que representa el acompañamiento en el estadio. Este cambio en el discurso no afirma que se deje por fuera el fervor y la pasión característica del Aguante, sino que fortalece los aspectos positivos del barrismo.

"Yo creo que, para ser barrista, es ese saber por qué estás ahí, por qué estás defendiendo a tu equipo, entonces si el equipo sacó a la venta acciones yo debo comprar acciones, debo saber qué es lo que se mueve, debo saber cómo funciona mi equipo, saber cómo funciona la barra. Sí, saber todo eso, el grupo de trapos, eso funciona así y asa, esas cosas, que lo van haciendo a uno un verdadero barra." (Héctor, comunicación personal, 16 de agosto de 2018).

"El aguante lo podemos mirar desde diferentes contextos, pero en pocas palabras, es la resistencia. La resistencia que yo tengo de cantar 90 minutos, de cantar y saltar 90 minutos derecho. El aguante que yo tengo 5 años en un descenso es algo realmente de aguante, el aguante que le tenemos que tener a un directivo, si esa constante resistencia. La hinchada utiliza mucho esa frase de aguante, porque digamos, el B.R. al ser la barra más estigmatizada, la que más represión le mandan, la que más, la más excluida, tales, lo que hacemos siempre es una cuestión de aguante, esa resistencia, estar ahí perseverando para impulsar al equipo hacía adelante." (Héctor, comunicación personal, 16 de agosto de 2018).

En suma, el Barrismo Social, invita a los sujetos a entender su identidad como barristas, a través de un conocimiento sobre la historia, que posibilita la existencia de la barra, y la consciencia sobre el papel que juegan los barristas para el equipo, y para la sociedad. 
De la misma forma, el proceso de barrismo social trajo consigo la transformación de una serie de prácticas y de elementos que son fundamentales para la identidad del barrista y que hasta ahora se han entrelazado con prácticas violentas, precisamente porque corresponden al Aguante.

No obstante, no todas las acciones ejercidas por FUNDP fueron bien recibidas por el resto de la barra, en una ocasión los integrantes de FUNDP lograron pasar un cierre de frontera establecido por la Policía, porque cumplían con un protocolo de viaje seguro para los integrantes, a diferencia de los buses comunes que usa la barra. Esta acción no fue bien recibida, en parte porque "las barras bravas son colectivos fuertemente organizados en torno a una estructura piramidal de jerarquías, rangos, asimetrías y roles bien definidos a partir de una distribución desigual de los recursos grupalmente valorados y de una aceitada división de trabajo interna." (Cabrera et al., 2016, pp. 9). Esto quiere decir, que los privilegios, por lo general son para los líderes de la barra, o al menos, son aquellos quienes están en la parte más alta de la jerarquía, quienes deciden cómo distribuir los incentivos o privilegios. Dado que, ninguno de los integrantes de FUNDP, se encuentra en la cúspide jerárquica de la barra, ni ostenta un rol que no sea el de ser barrista de Barón Rojo se generaron fuertes tensiones con el resto de la barra.

"Eso generó un recelo dentro de la barra, y la barra optó por excluir al bloque un tiempo, no nos daban boletería, ni nada de eso de la barra" (Héctor, comunicación personal, 16 de agosto de 2018).

La situación se tornó cada vez más complicada, pues en un viaje a Bogotá en un partido contra Santa Fe, el Barón Rojo estaba esperando el bus en el que venía la fundación y los integrantes del bloque, el cual era un bus de una empresa oficial, para atacarlo con piedras. 
"Por cosas de la vida, sacamos el bus de aquí y nos fuimos para la terminal de Cali, y nos pasamos de bus, cambiamos a un Velotax. Entonces, cuando llegamos allá a Bogotá ellos quedaron despistados, como que estaban esperando un Flota Magdalena, pero vieron llegar fue un Velotax, entonces no lo lograron pues, quebrarle los vidrios ni nada" (Héctor, comunicación personal, 16 de agosto de 2018).

En ocasiones, las prácticas que se fomentan desde el barrismo social interfieren con las mafias que utilizan a Barón Rojo como una plataforma de expendio de drogas, con las dinámicas de ilegalidad que ven en la barra un espacio donde la ley no rige de la misma manera que en el resto de la ciudad y contra los discursos del Aguante.

"Porque pues ellos denunciaron todo el tema del micro-tráfico que había dentro de la barra, ellos lo denunciaron a las actividades, hubo capturas, y toda esa olla se destapo, como quien dice, ya los medios ahí, por eso es que en ese caso a ellos los asesinan" (Héctor, comunicación personal, 16 de agosto de 2018).

"De ahí realmente fue que surgieron los problemas, porque toda la gente que ya venía acostumbrada a un chip, a un modelo de la barra más beligerante, y temas delincuenciales pues ya empezaron a ser la piedra en el zapato, porque los temas sociales siempre van a trocar los intereses oscuros que haya" (Héctor, comunicación personal, 16 de agosto de 2018).

Sin embargo, la fundación mantuvo sus actividades a pesar de las amenazas recibidas, hasta que, en mayo de 2017, durante el clásico América - Deportivo Cali, que contó con la presencia de las dos hinchadas en el estadio. Se generó una batalla campal en la gramilla del Pascual Guerrero al finalizar el partido. Este suceso generó 
reacciones fuertes de parte de entidades gubernamentales, como la Alcaldía de Cali, que cortó temporalmente cualquier relación con la barra, y sancionó fuertemente al equipo, al Barón Rojo Sur y al Frente Radical Verde. En medio de esta desarticulación institucional, FUNDP mantuvo, por la confianza que había creado durante meses atrás, las relaciones con la personería municipal.

"Nuestro contrapeso a eso fue hacer el taller de barrismo social, con la universidad cooperativa y con la personería municipal, y estuvimos en el taller de barrismo social y explicamos la normativa del fútbol, el código de policía que estaba próximo a ser vigente, y el código de infancia y adolescencia y empezamos a explicar esas dinámicas a los integrantes de la barra, entonces, las dependencias de la Alcaldía cuando querían hacer un trabajo de barras no buscaban a las barras sino que nos buscaban a nosotros" (Héctor, comunicación personal, 16 de agosto de 2018).

De esta manera, FUNDP se convirtió en la cara positiva de la barra, y en Barón Rojo, los líderes se vieron en la necesidad de aceptar que esos procesos de barrismo social también eran parte de Barón Rojo, y que trabajando en esa línea podían mejorar la relación con aquellas entidades que los pueden perjudicar.

"La barra se dio cuenta digamos de eso, y ya fue que ellos luego sientan como cabeza y empezamos a hacer un proceso de paz entre nosotros, y ahora las cosas van bien" (Héctor, comunicación personal, 16 de agosto de 2018).

\section{Barrismo Social sin colores.}

La visión sobre el barrismo social se ha transformado. Pues, según decreto 1007 de 2012, barrismo social son "Acciones en- 
caminadas a redimensionar las formas de expresión y las prácticas de los integrantes de las barras de fútbol" (Decreto 1007, 2012) sin determinar que la ejecución del barrismo por parte de integrantes de una barra deba dirigirse exclusivamente a los integrantes de la misma barra. Por ello, FUNDP ha realizado acciones en conjunto con miembros del Frente Radical Verdiblanco, e incluso con procesos de barrismo social gestados desde la barra del Independiente Medellín, Rexixtenxia Norte.

"Como fundación se nos amplió la visión y cuando hicimos las paces con la barra eso fue lo que más claro le dejamos, en este momento nuestra apuesta es el barrismo social. Somos americanos y somos barón rojo y eso son códigos internos que uno lleva, y en el actuar de uno se sabe, uno no tiene que estar vociferando, ni mostrando los tatuajes ni nada, pero ya en nuestro actuar, como nuestra responsabilidad con el fútbol, desde que creamos la fundación ya nos sentimos responsables con la transformación del fútbol y de las hinchadas acá en Colombia. La visión se nos amplió a trabajar con cualquier tipo de fundación o con cualquier hinchada, y no solo barra brava" (Héctor, comunicación personal, 16 de agosto de 2018).

Es claro, que las acciones de la fundación están enmarcadas bajo los referentes identitarios e ideológicos propios del barrismo. Razón por la cual, una de las formas de tomar distancia por parte de los miembros de FUNDP fue a través de uno de los elementos que más valor tienen para la barra, los trapos.

"Nosotros hicimos un trapo y ese trapo generó bastante controversia dentro de la barra, nosotros hicimos este trapo, ese fue el que nos amplió la visión a decir realmente podemos convivir todos los colores dentro de este mismo país porque tenemos un enemigo en común, yes esteque siempreva aestar ahí presionándolos, paraque 
nunca salgamos adelante, para que siempre sigamos, como muchos dicen en su discurso, una manada de gamines, muchos dicen eso pero siempre le apuestan a que eso siempre siga siendo así, y ese es el enemigo en común y al que realmente las barras tenemos que trabajar articuladamente en esa transformación" (Héctor, comunicación personal, 16 de agosto de 2018).

Imagen 1. Trapo Por un mejor país fútbolero.

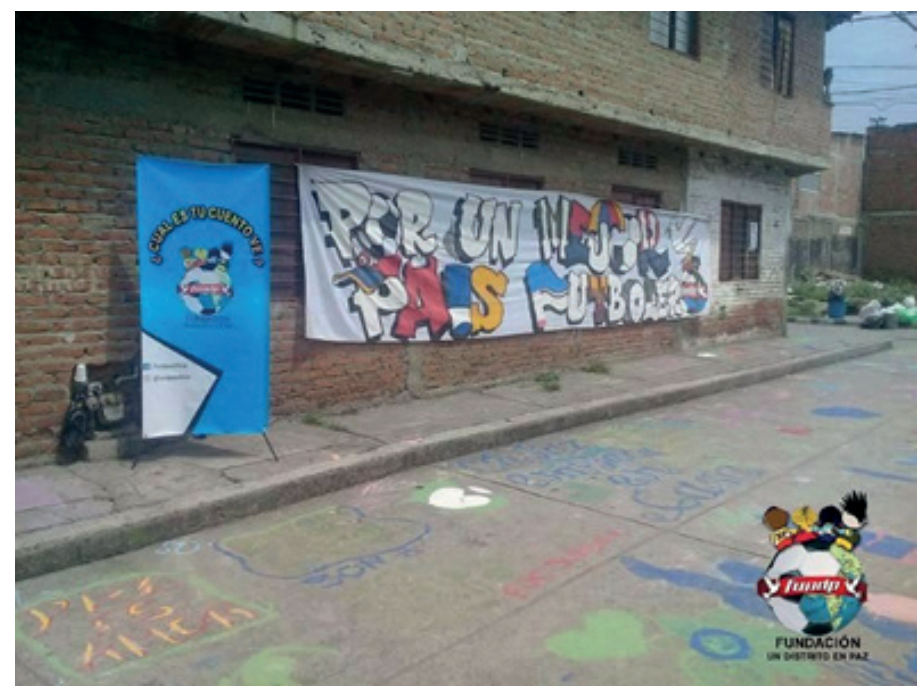

Fuente: Elaborado por Fundación Un Distritoen Paz

Como se puede evidenciar, el trapo, a diferencia de los trapos utilizados por la barra que son principalmente de los colores alusivos a ésta, tiene los colores de varios de los equipos del país, con el fin de evidenciar que el barrismo social es un proceso que trasciende los colores de cada barra o de cada equipo. Este trapo es fundamental para la fundación, pues para los barristas "la identidad de la barra está en los trapos, ya que los jóvenes ven reflejados en estos la manera de representarse y ser identificados por 
la sociedad." (Quintero y Delgado, 2013, pp. 85). En este orden de ideas, el hecho de que los integrantes de FUNDP se sientan recogidos en este trapo, permite ver el uso que le han dado a un elemento tan polémico y determinante para las barras, como los trapos. El trapo, involucra elementos simbólicos que marcan una distancia de la barra y plantea como postura de FUNDP que el barrismo social no puede discriminar por colores, ni rivalidades.

\section{(iv) Reflexiones en torno al Barrismo Social}

Teniendo en cuenta que el Barrismo Social no ha sido ampliamente trabajado por la academia, el presente artículo pretende sentar una serie de elementos, que se consideran pertinentes para una futura agenda de trabajo en torno a este concepto.

El caso de Barón Rojo y FUNDP permite ver como las prácticas características de la identidad de los barristas, que se ha dicho que son insumos para la legitimidad y la ejecución de la violencia, también, pueden convertirse en elementos útiles para el desarrollo de procesos positivos que aporten la individualidad de los barristas y a la construcción de un consciencia social y política. Por ende, los procesos de barrismo social pueden ser entendidos como procesos de empoderamiento sobre la identidad, en este caso sobre la identidad del barrista, pues se apela a elementos como el conocimiento de la historia sobre la barra y el equipo, y al reconocimiento de lugar y de la función social que cumple la barra para la sociedad.

De igual forma, el barrismo social puede ser entendido como una metodología de intervención social, debido a que ofrece herramientas de pedagogía que son atractivas para los jóvenes, niños y niñas de la sociedad colombiana, como, por ejemplo, fomentar valores cívi- 
cos y sociales, a través del fútbol que puede ser un deporte cercano para ellos. De esta misma manera, el barrismo social apunta a las construcciones de una subjetividad política y de una ciudadanía.

Se resalta su papel metodológico, pues no solamente se dirige a una población perteneciente a las barras, sino a quienes pueden tener cercanía. Además, en contextos donde se carece de oportunidades, hay condiciones de marginalidad y el reconocimiento social se convierte en una necesidad insatisfecha para la construcción de la identidad, la barra se presenta como una alternativa. Si bien en algunos casos la alternativa se entrelaza con prácticas violentas, el fin del barrismo es posicionar como discurso predominante, y sistema de prestigio y honor, el barrismo social. Sumado a todo lo anterior, la desarticulación de la fundación de la barra, y la ampliación de su visión como organización, permite ver que la definición de barrismo social ofrecida en la ley podría ser ampliada, apuntando a identificar en estos procesos, objetivos que trascienden a los barristas como sujetos de intervención, y situarse en un contexto mucho más amplio. Por consiguiente, se considera pertinente el siguiente apartado.

Hoy en día, hablar de Barrismo Social es referirse a un proyecto social y político, cuyo propósito más amplio consiste en transformar las relaciones de desigualdad, exclusión y las prácticas con contenidos de violencia, en medio de las cuales constituyen su personalidad los niños, niñas y jóvenes en condiciones de vulnerabilidad y asociados a los parches y barras futboleras; lo anterior, otorgándole un papel fundamental y responsable a quienes hacen parte de estas formas de organización social y protagonizan sus acontecimientos cotidianos (Castillo, 2013).

El fragmento citado, corresponde a lo que apunta FUNDP, puesto que finalmente los proyectos apuntan, a la transformación de la 
relación de desigualdad y exclusión que se dan asociados al territorio, en el que se encuentra el Bloque Distrito Popular, al que pertenecen. También, han participado o apoyado procesos de manifestación social en pro de la educación pública, pues reconocen que la falta de oportunidades es lo que genera marginalidad en su territorio, y en otros lugares, a nivel nacional, en donde también existe el barrismo. Por tanto, al construir una ciudanía activa, que incide y participa social y políticamente de manera positiva, lo que se genera es una continuidad de los procesos de barrismo social, que se ejecutan desde la noción e identidad de los barristas. Contrario a lo que sucede con las acciones del aguante tradicional que crean un círculo de violencia, el barrismo social y el aguante-social crean un ciclo de procesos positivos para la barra y para la sociedad.

\section{Conclusión}

Finalmente, a manera de conclusión puede decirse que la barra B.R.S. no es un actor monolítico, sino que está compuesta por sujetos provenientes de contextos y realidades diferentes. Por ende, da lugar a procesos políticos y sociales, como los realizados por Barón Rojo y FUNDP, que evidencian la necesidad de repensar si el concepto de Aguante puede explicar la realidad de esta barra. De esta manera, a nivel nacional esta característica de las barras ha tenido un reconocimiento normativo en la ley 1270 de 2009, el decreto 1007 de 2012 y el Plan Decenal de Seguridad, Comodidad y Convivencia en el Fútbol (PDSCCF) a través de un concepto, que podría llenar el vacío que hay en la literatura sobre la comprensión de las acciones de las barras colombianas, Ilamado barrismo social.

Posterior a este reconocimiento normativo, en Barón Rojo surgieron procesos como los de FUNDP que tuvieron un marco en el 
cual apoyarse para consolidar y formalizar sus procesos sociales y políticos, trascendiendo los colores y símbolos de una barra, y ampliando el significado de barrismo social a un proceso de lucha contra la exclusión y la marginalidad.

De igual forma, se reconoce que se carece de la sufriente profundidad teórica sobre el concepto de barrismo social, por ende, se sientan cuatro reflexiones que puedan aportar a un futuro desarrollo teórico de este. El barrismo social (i) Puede ser entendido como un proceso de empoderamiento sobre la identidad. (ii) Puede usarse como una metodología de intervención social atractiva para los jóvenes en contextos donde hay grandes cantidades de barristas. (iii) Transforma el discurso tradicional de aguante hacía un enfoque social y político en el que el reconocimiento y reputación de la barra se consigue por medio de otros elementos distintos a la violencia, que generan formas de ciudadanía activas y participativas. (iv) Trasciende las dinámicas o problemáticas de la barra y se convierte en un proyecto social y político con características democráticas (Castillo, 2013). 


\section{Bibliografía}

Alcaldía de Cali (2017). Caracterización de las barras de fútbol Frente Radial Verdiblanco-FRV y Barón Rojo Sur -BRS en la ciudad de Santiago de Cali. Cali.

Aponte, D., Pinzón, D., Rodríguez, D. \& Vargas, A. (2008). Las barras de fútbol en Colombia: balance de la producción académica y algunas reflexiones sobre su cubrimiento periodístico, programas y normatividad (2000 - 2008). CERAC.

Archetti, E. (2008). El potrero y el pibe. Territorio y pertenencia en el imaginario del fútbol argentino. Horizontes Antropológicos, pp.259-282.

Arroyo, C. (2014). Barrismo social y colectivo barrista colombiano: los antecedentes del diseño de una política pública. Pregrado. Universidad del Valle.

Barra Brava (2019). Historia de la barra brava Barón Rojo Sur y la hincha del club de fútbol América de Cali de Colombia. [Blog] Barra Brava.

BBC News Mundo. (2019). Cómo surgieron los "hooligans", Ios violentos aficionados al fútbol inglés. BBC. [online] disponible en: https://www.bbc.com/mundo/noticias/2015/01/150122_deportes_historia_hooligans_finde_yv [Accedió 2 Mar. 2019].

Cabrera, N., Czesli, F. y Garriga, J. (2016). El aguante en debate: violencia en el fútbol y políticas públicas en la Argentina. Esporte e Sociedade.

Carrión, F., y Rodríguez, M. (2014) Luchas urbanas alrededor del fútbol. Ecuador: 5ta avenida editores. 
Castillo, A. (2013). Barrismo social: primer acercamiento a una definición. Estrategias para la participación en la formulación del Plan Decenal de Seguridad, Comodidad y Convivencia 2013-2123 en el Fútbol, desde el Barrismo Social. Bogotá: Coldeportes.

Castro, J. (2010). Etnografía de hinchadas en el fútbol: una revisión bibliográfica. Maguaré, pp.131-156.

Clavijo, J. (2011). Estudio de Barras de Fútbol De Bogotá: Los Comandos Azules. Prácticas sociales y construcción de la identidad urbana, pp.42-59.

Congreso de la República de Colombia. (5 de enero de 2009) Estatuto del Aficionado al Fútbol en Colombia. [Ley 1279 de 2009]. DO: 47.223.

Congreso de la República de Colombia. (16 de mayo de 2012) Estatuto del Aficionado al Fútbol en Colombia. [Decreto 1007]. DO: 48.432.

Elías, N. and Dunning, E. (1992). Introducción. en: N. Elías and E. Dunning, ed., Deporte y Ocio en el proceso de civilización. Madrid: Fondo de Cultura Económica, pp.31 - 81.

Elías, N. and Dunning, E. (1996). La búsqueda de la emoción. en: N. Elías and E. Dunning, ed., Deporte y Ocio en el proceso de civilización. Madrid: Fondo de Cultura Económica, pp.83 - 115.

Garriga, J. (2006). "Acá es así": Hinchadas de fútbol, violencia y territorios. Avá, Revista de Antropología, 93 - 107.

Gómez, S., y Opazo, M. (2008). Características estructurales de un club de fútbol profesional de elite. IESE Business SchoolUniversidad de Navarra. 
Halbwachs, M. (2004). Memoria colectiva y memoria histórica. En: M. Halbawchs, La memoria colectiva. España: Prensas Universitarias de Zaragoza, 53 - 88.

Londoño, A., y Pinilla, V. (2009). El Barrismo Social de Hinchas por Manizales. Una práctica política y ciudadana. Revista Austral de Ciencias Sociales, pp. 16 - 73.

Máximo, C. (2014). El Fútbol: construcción de múltiples identidades en conflicto. En Carrión, F., \& Rodríguez, M. Luchas urbanas alrededor del fútbol. (pp. 243 - 275). Ecuador: 5ta avenida editores.

Ministerio del Interior (2014). Plan Decenal de Seguridad, Comodidad y Convivencia en el Fútbol. Colombia.

Ministerio del Interior (2014). La gran encuesta del fútbol en Colombia: El poder del Fútbol. Bogotá D.C.

Ramírez, J. and Serrano, J. (2014). Hinchas, territorios y violencia en el fútbol ecuatoriano. Esporte e sociedade, 1-17.

Rojas, L. (2013). Las barras bravas como una tribu urbana. Una búsqueda de identidad. Estudio de caso del Barón Rojo Sur y Frente Radical Verde. Pregrado. Universidad del Valle.

Villana, S. (2014). El fútbol, territorio (local, nacional, global) de pasión y de tedio. In: F. Carrión and M. Rodríguez, ed., Luchas Urbanas alrededor del fútbol. 5ta Avenida Editores, pp.313 - 340. 